\title{
Reduced order modeling of some fluid flows of industrial interest
}

\author{
D Alonso, F Terragni, A Velazquez and J M Vega \\ E.T.S.I. Aeronáuticos, Universidad Politécnica de Madrid, 28040 Madrid, Spain \\ E-mail: josemanuel.vega@upm.es
}

\begin{abstract}
Some basic ideas are presented for the construction of robust, computationally efficient reduced order models amenable to be used in industrial environments, combined with somewhat rough computational fluid dynamics solvers. These ideas result from a critical review of the basic principles of proper orthogonal decomposition-based reduced order modeling of both steady and unsteady fluid flows. In particular, the extent to which some artifacts of the computational fluid dynamics solvers can be ignored is addressed, which opens up the possibility of obtaining quite flexible reduced order models. The methods are illustrated with the steady aerodynamic flow around a horizontal tail plane of a commercial aircraft in transonic conditions, and the unsteady lid-driven cavity problem. In both cases, the approximations are fairly good, thus reducing the computational cost by a significant factor.
\end{abstract}

\section{Introduction}

Instabilities and bifurcations are common in fluid flows of industrial interest and will be (sooner or later) unavoidably relevant in the design and certification of industrial devices. Instabilities may have a detrimental effect on either safety (e.g. flutter instability may produce large vibrations in aircraft) or performance (e.g. laminar/turbulent transition increases skin friction in boundary layers), but they can also be beneficial (to, e.g., promote convection in micro-heat exchangers, see Meis et al (2010)). The required bifurcation analysis should include not only the instability limits but also the whole (or at least a part of the) bifurcation diagram, which usually requires a great deal of computational effort, not compatible with the strict cost and time constraints that are common in the industry. For instance, calculation of the aerodynamic flow around a commercial aircraft is not possible today using direct numerical simulation, and requires several CPU days using the so-called high fidelity solvers based on turbulence modeling. Thus, aerodynamic calculations in the conceptual design of commercial 
aircraft are currently based on either semi-empirical formulae or quite low fidelity models, which must be tested/corrected by experimental tests.

Reduced order models (ROMs) may help us to fill this gap. A class of ROMs is based on the projection of the governing equations onto a low-dimensional manifold. This can be determined by applying proper orthogonal decomposition (POD) to a set of previously calculated snapshots that should be representative of the considered flow patterns. The snapshots can be obtained using a computational fluid dynamics (CFD) solver on the full set of governing equations. The method can be applied to both steady and time-dependent problems, which will be considered in sections 2 and 3 , respectively. To obtain robust, computationally efficient ROMs requires attending to several difficulties/opportunities, in connection with, e.g., the following.

- The inner product that is used to project the governing equations, which can be based on the whole computational domain, but also on a limited number of mesh points only,

- The equations that are actually projected, which can be either the exact governing equations or the equations that are used by the CFD solver (numerical artifacts included),

- The use of industrial/open source CFD solvers, whose details are not known,

- The role of CFD errors.

Several basic ideas are presented in connection with these difficulties, taking advantage of the opportunities. For illustration, two examples are considered that result from steady, transonic aerodynamics (section 2) and the unsteady lid-driven cavity (section 3 ). The paper ends with some concluding remarks in section 4 .

\section{Steady ROMs}

Steady ROMs of nonlinear, multi-parameter problems require us to combine reduced order modeling with iterative methods to localize the steady states. A few ROMs for steady problems are available in the literature, after the work of LeGresley and Alonso (2001), Rouizi et al (2009), (Alonso et al 2009a, 2009b, 2010) and Bache et al (2010, 2012). Here, we summarize the basic method presented and fully discussed by Alonso et al (2009). The following steps are shared by all the variants/versions.

(i) A set of $N_{0}$ steady states (known as snapshots), $\boldsymbol{q}_{1}, \ldots, \boldsymbol{q}_{N_{0}}$, is calculated using a CFD solver for $N_{0}$ sets of parameter values.

(ii) POD is applied to the set of snapshots, to obtain a set of POD modes, $\boldsymbol{Q}_{1}, \ldots, \boldsymbol{Q}_{N_{0}}$.

(iii) The solution is written down as a linear combination of the $n$ most energetic POD modes, $q \simeq \sum_{k=1}^{n} A_{k} Q_{k}$, where $n$ is selected using the standard a priori error estimate associated with POD.

(iv) The POD-mode amplitudes are calculated minimizing a properly defined residual based on the equations and boundary conditions.

The first three steps are performed offline, to construct the ROM, and the last step is performed online to calculate steady states for each set of parameter values. Additional ingredients to improve computational efficiency include the following.

a. Either independent POD modes for each flow variable or joint modes can be defined.

b. Both POD and the residual minimization can be performed using a limited number of mesh points (say, twice the number of retained modes), which can be concentrated in a projection window. Appropriate selection of the projection window excludes localized CFD errors. 
c. The residual can be based on either the exact Navier-Stokes equations or the equations implicit in the CFD solver. At very large Reynolds number, the Euler equations can be used to define the residual, which makes the method independent of the peculiarities of the CFD solver, such as turbulence modeling.

d. The projection window can be divided into several regions, in which POD is applied independently.

e. The POD manifold can be defined locally in the parameter space.

f. Steep spatial gradients (e.g. shock waves in transonic aerodynamic flows) may require specific treatment (Alonso et al 2010).

g. Variable geometry can be accounted for using a common, virtual mesh to define POD modes (Bache et al 2010). The virtual mesh is smoothly mapped back and forth onto the actual meshes, where the residual is minimized.

$\mathrm{h}$. The residual minimization can be performed using either a genetic algorithm, a gradientlike method or a combination of both, and can be combined with a continuation method. The genetic algorithm is generally more robust and able to search a significant part of the phase space. It can be fairly standard (emphasizing the robustness of the process); see Alonso et al (2009b, 2010, 2012), for the selection of the various tunable parameters of the genetic algorithm. Gradient-like methods are much faster but require a good initial guess, which can be provided using a continuation method in the parameter space. A gradient-like method that yields good results in this context is a quasiNewton, Broyden-Fletcher-Goldfarb-Shanno method, see Bache et al (2012) for further details.

Item (c) can be surprising at first sight, since the Euler equations themselves are not appropriate for simulating detached aerodynamic flows, exhibiting significant recirculation bubbles and wakes. Moreover, as will be illustrated below, the ROM provides good approximations of the flow variables in both the detached regions and the wakes, and even in the boundary layers (where the ignored viscous effects are dominant). To understand this requires noting that the CFD calculated snapshots already contain the correct vorticity distributions on the boundary layers and the wakes. This information is inherited by the POD modes. The ROM only needs to select the correct values of the mode amplitudes moving in a low-dimensional manifold, and this can be made using information from nearly inviscid regions of the flow field.

The resulting ROM has been applied to a number of steady state problems resulting from micro-fluidics and aerodynamics, obtaining quite good results in a robust and computationally efficient way. For illustration, we consider here the aerodynamic flow around a commercial aircraft horizontal tail plane (HTP), for varying values of the angle of attack $\left(-3^{\circ} \leqslant A \circ A\right.$ $\left.\leqslant 3^{\circ}\right)$ and Mach number $(0.4 \leqslant M \leqslant 0.8)$. In this parameter range, the aerodynamic flow presents fairly strong shock waves, but these move only slightly as the parameters are varied. This means that no special shock wave treatment is needed. Snapshots are calculated using an industrial CFD code based on a finite-volume discretization (Tannehill et al 1997) of the compressible continuity, momentum and energy equations, with viscosity terms modified according to an Edwards-corrected (Edwards and Chandra 1996) Spalart-Allmaras turbulence model (Spalart and Allmaras 1992) and some terms added for numerical reasons; see Alonso et al (2012) for further details of the CFD solver.

The span length of the HTP is 1.5 times its root chord, $c$. The computational mesh (figure 1, left) is structured, with a total number of 3053744 points in a parallelepipedic computational domain of sides $-5 c<x<10 c, 0<y<10 c$ and $-10 c<z<10 c$. Those blocks that are adjacent to the HTP surface build which will be called the O-mesh (figure 1, 

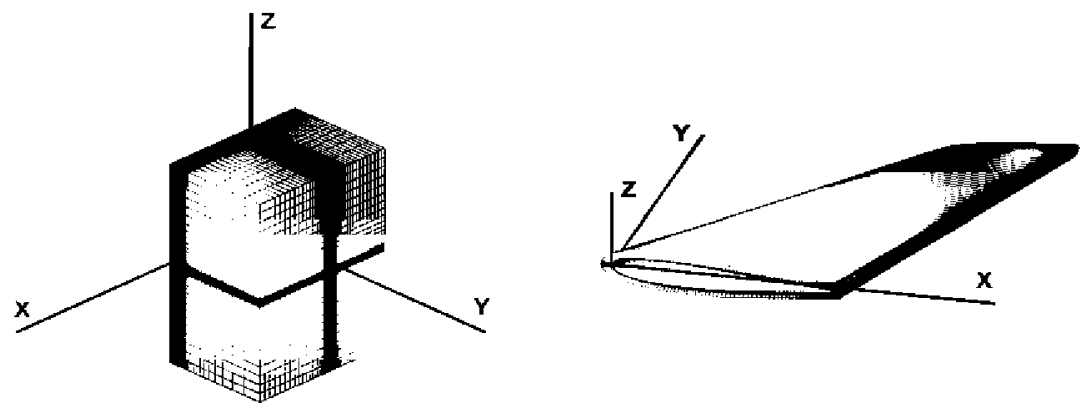

Figure 1. Three-dimensional views of the whole computational mesh (left) and the HTP with the O-mesh (right).

Table 1. rms errors (in \%) in the O-mesh of the flow variables for the three combinations of snapshots; rms errors in the whole computational mesh are given in parentheses.

\begin{tabular}{lccc}
\hline Combination & 1 & 2 & 3 \\
\hline$\rho u$ & $0.38(0.01)$ & $0.60(0.03)$ & $1.02(0.03)$ \\
$\rho v$ & $0.40(0.00)$ & $0.60(0.00)$ & $0.93(0.00)$ \\
$\rho w$ & $0.18(0.14)$ & $0.22(0.25)$ & $0.31(0.27)$ \\
$\rho$ & $0.23(0.01)$ & $0.30(0.02)$ & $0.40(0.02)$ \\
$p$ & $0.25(0.01)$ & $0.33(0.02)$ & $0.42(0.02)$ \\
\hline
\end{tabular}

right), whose wall normal size is comparable to the HTP thickness. The O-mesh contains 840825 points.

The basic method and the additional ingredients mentioned above are used in the following manner (see Alonso et al (2012) for further details). The residual is based on the Euler equations, and defined using a part of the $\mathrm{O}$-mesh as a projection window (excluding the boundary layers attached to the wall). The $\mathrm{O}$-mesh is divided into two regions, the pressure and suction sides of the HTP, and only 104 mesh points scattered in each region are used to define the residual; in addition, six points in the upstream far field are used to account for the free stream boundary conditions. Joint POD modes are defined locally in the parameter space, using only the nearest snapshots (with an appropriate definition of distance among snapshots) in each calculation; the number of snapshots required to obtain the POD manifold is selected by the method. The results below are calculated using three combinations of snapshots, forming three structured, equispaced grids in the $M \times A o A$ plane, which will be used to calculate the POD manifold. These will be called combinations \# 1, \# 2 and \# 3, and contain $9 \times 13=117,7 \times 5=35$ and $5 \times 5=25$ snapshots, respectively.

Table 1 shows the root mean square (rms) errors in the ROM-reconstructed flow variables, both in the $\mathrm{O}$-mesh and in the whole computational domain, averaging results in 28 representative test points scattered in the $M \times A o A$ plane. Note that the rms errors in the $O$-mesh are always larger than their counterparts in the whole computational domain, which is consistent with the fact that the largest flow activity occurs precisely in the O-mesh. The errors in the whole computational domain are of the order of $10^{-3}$, which is the value of the POD truncation error that was used to select the numbers of retained modes in the three combinations of snapshots. In any event, the rms errors are always below $1 \%$ for the three snapshot combinations, which is more than enough in most industrial applications. 

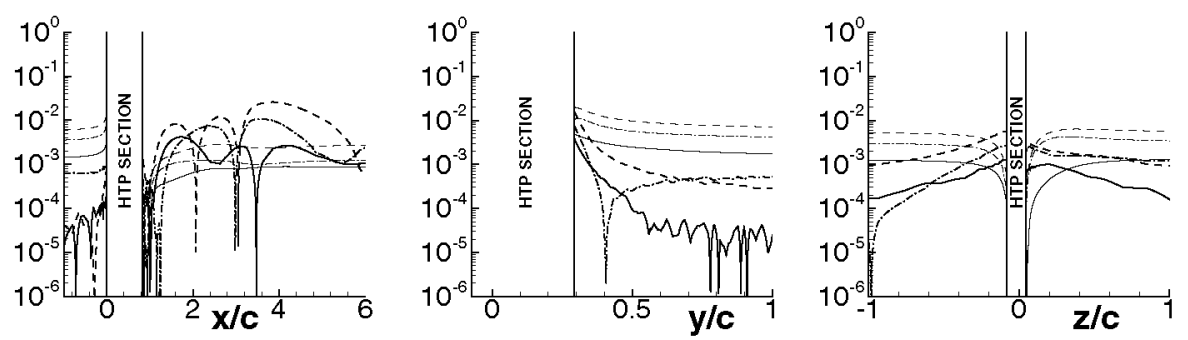

Figure 2. Difference between the CFD and ROM values for $\rho u$ and $\rho w$ (thick and thin lines, respectively) along the lines $y=0, z=0$ (left), $x=0, z=0$ (center) and $x=0, y=0$ (right) at $(M, A o A)=\left(0.775,2.25^{\circ}\right)$. ROM results are calculated using the snapshot combinations 1 (solid lines), 2 (dot-dashed lines) and 3 (dashed lines).
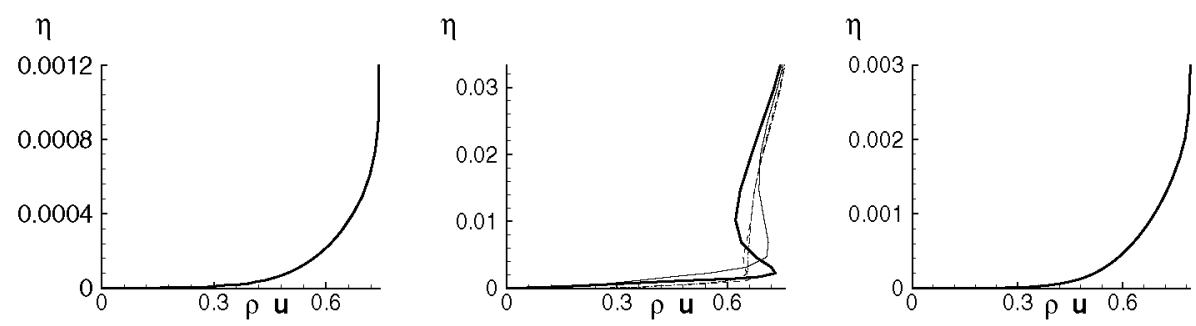

Figure 3. Streamwise velocity distribution along the wall normal coordinate in the boundary layer at three representative points in the HTP surface, at $(M, A \circ A)=\left(0.775,2.25^{\circ}\right)$, as calculated using CFD (-) and ROM results considering the snapshots combinations 1 (-), 2 (--) and $3(--)$.

In order to illustrate local approximations, the distributions of the streamwise and spanwise fluxes along the axes of the Cartesian coordinate system, at $(M, A O A)=$ $\left(0.775,2.25^{\circ}\right)$, are shown in figure 2 . Note that, as expected, the errors decrease as the number of snapshots increases. Combination 1 provides errors of the order of $10^{-3}$, and the coarser combination 3 still provides errors of the order of $10^{-2}$, even in the wake (left plot, downstream of the HTP section). These are more than reasonable, and somewhat surprising at first sight, since both the POD and the residual calculation have been done using information from the projection window (which is contained in the $\mathrm{O}$-mesh) only. The reason for such good results is that the wake is somewhat slaved to the flow field upstream of the HTP, due to redundancies that are accounted for in the snapshots, and recognized by POD. Thus, the approximation in the wake will be good provided that the upstream flow is well calculated.

Similarly, the reconstruction of the aerodynamic field in the boundary layer near the HTP surface is illustrated in figure 3 , where the streamwise velocity along the wall normal direction is plotted for three representative points of the HTP surface, at $(M, A o A)=\left(0.775,2.25^{\circ}\right)$; the left and right plots correspond to generic points, but the middle plot to a point that is precisely below a shock wave. Note that the results are quite good in the right and left plots since CFD and ROM results are plot-indistinguishable, even with the coarser snapshots combination 3; the associated errors are similar to those in figure 2, namely of the order of $10^{-2}$ and $10^{-3}$ for combinations 3 and 1 , respectively. As expected, results worsen near shock waves (middle plot). As happened with the approximation in the wake, the ROM results are so good near any generic point of the HTP because the boundary layer structure is somewhat slave (along the wall normal direction) to the outer flow. Thus, if the latter is well calculated 
(which occurs if no nearby shock wave is present), then the boundary layer must also be well approximated.

Concerning the computational cost, each CFD run requires 3 CPU hours, and each ROM calculation needs 10 CPU minutes if the residual is minimized with a genetic algorithm, and 3 CPU seconds if a gradient-like method is used.

\section{Time-dependent ROMs}

Time-dependent ROMs based on POD were introduced almost 20 years ago for the fast simulation of incompressible fluid flows exhibiting complex dynamics (Berkooz et al 1993). Since then, an increasing number of works on the topic appeared in the literature approaching problems of scientific and industrial interest, taking advantage of the ability of such ROMs to drastically reduce the computational cost associated with the involved numerical simulations (Dowell and Hall 2001, Rempfer 2003, Lucia et al 2004, Lieu et al 2006, Bizon et al 2008, Thomas et al 2010). Unsteady ROMs are also used to speed up the calculation of steady states when these are computed as attractors at large times. Still the major challenge in the field is developing effective ROMs to cope with turbulent flows.

When applied to a parabolic, dissipative system of the form

$$
\mathcal{M} \partial_{t} q=\mathcal{L} q+f(q, t)
$$

where $\mathcal{M}$ (positive definite) and $\mathcal{L}$ (elliptic) are linear operators (with the highest-order derivatives accounted for in $\mathcal{L}$ ), and $f$ is a nonlinear operator, these ROMs consist in (i) CFD-computing some flow snapshots from (1), (ii) applying POD and extracting the $n$ most energetic POD modes (selected using standard POD formulae), (iii) expanding the state variable as $\boldsymbol{q} \simeq \sum_{i=1}^{n} A_{i}(t) \boldsymbol{Q}_{i}$ and (iv) Galerkin projecting (1) onto the POD manifold which yields a Galerkin system (GS), which is a low-dimensional ROM of the given ordinary differential equation (ODE) system in the time-dependent unknown amplitudes $A_{i}$ ).

The snapshots should be representative of the dynamics to be approximated; that is, they must span a portion of the involved dynamical system's phase space containing all relevant orbits (Sirovich 1987). Thus, most of the existing POD-based ROMs are generally suitable for simulating attractors but not transient behaviors. The latter requires adaptive methods (Ravindran 2000, Bergmann and Cordier 2008, Rapún and Vega 2010, Tadmor et al 2010, Terragni et al 2011). POD modes can be calculated either from the outset (preprocessed POD plus Galerkin projection methods) or updated during the time integration of the GS (Bergmann et al 2009, Rapún and Vega 2010, Terragni et al 2011). The projected nonlinearity needs (in principle) to be computed at each time step. Nevertheless, when $f$ is algebraic (as, e.g., in the Navier-Stokes equations), its projection onto the POD modes is a polynomial in $A_{1}, \ldots, A_{n}$ whose coefficients may be calculated in a preprocess. This would be faster than performing the mentioned projection, but determining the polynomial coefficients could offset such an advantage when many projections must be carried out. On the other hand, the computational efficiency of the GS construction can be improved by considering a nonstandard inner product in the POD manifold (Alonso et al 2009, 2010, Bache et al 2010, Rapún and Vega 2010, Terragni et al 2011).

A major difficulty related to time-dependent POD-based ROMs is that truncated GSs may exhibit spurious dynamics in a somewhat unpredictable way. In some cases, this seems to be due to the noninvariance of the POD manifold under the true dynamics (Rempfer 2000). A variety of techniques intend to handle this high-order modes truncation instability by correcting either the GS or the POD manifold to make the latter invariant. Some examples are the introduction of additional terms in the GS (Sirisup and Karniadakis 2004, 


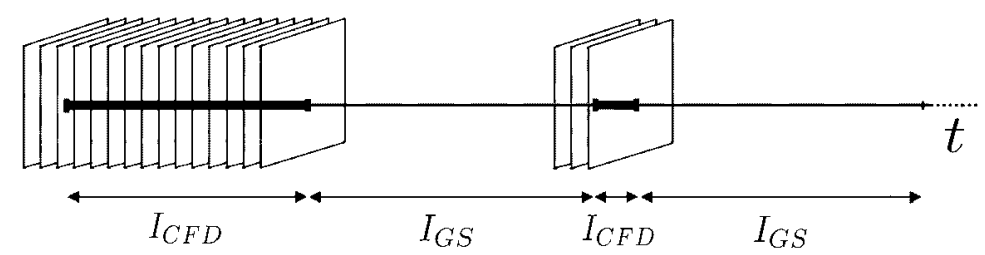

Figure 4. The LPOD+GP method. Snapshots are CFD-computed in each $I_{\mathrm{CFD}}$ interval and used to either calculate (in the first $I_{\mathrm{CFD}}$ interval) or update (in subsequent $I_{\mathrm{CFD}}$ intervals) some POD modes. Two GSs based on (different numbers of) these modes are integrated in the $I_{\mathrm{Gs}}$ intervals.

Couplet et al 2005, Sirisup et al 2005), the combination of the GS with a CFD solver (Sirisup et al 2005, Bergmann et al 2009), the comparison of the solutions of two GSs retaining different numbers of POD modes (Rapún and Vega 2010, Terragni et al 2011) and the use of residual modes (Bergmann et al 2009). In this context, the open flow around a cylinder was considered by some authors as a benchmark problem to identify a means of stabilizing POD-based ROMs (Noack et al 2003, Siegel et al 2003, 2008, Tadmor et al 2010).

The method introduced in Rapún and Vega (2010) represents a somewhat different approach to the field, resulting from a critical view of the POD-based reduction techniques. The local POD plus Galerkin projection (LPOD+GP) method shares a few ingredients with some of the adaptive methods cited above, but exploits new ideas based on two main observations. Namely, (a) when dealing with transient dynamics, POD manifolds calculated from snapshots in a time interval $t_{0}<t<t_{1}$ are also appropriate for larger values of $t$, provided that some additional POD modes are retained (in other words, the parabolic nature of (1) reflects in the time-continuity of the POD manifold); (b) truncation instability can be detected by comparing the high-order modes behavior of two GSs retaining different numbers of POD modes.

The LPOD+GP method allows us to construct robust, adaptive POD-based ROMs for generic dissipative systems. It has been tested to efficiently simulate highly unstable transient dynamics, without any numerical instability (Rapún and Vega 2010, Terragni et al 2011). The method (figure 4) is based on the combined use of a CFD solver, run to compute snapshots, and two GSs in interspersed time intervals, $I_{\mathrm{CFD}}$ and $I_{\mathrm{GS}}$, respectively. It aims to approximate a solution of (1) within a desired error-bound $\varepsilon$ in each $I_{\mathrm{GS}}$ interval. The following elements are an essential overview of the method (see Rapún and Vega (2010) and Terragni et al (2011) for further details).

Consistent with the remarks above, the POD manifold is completely constructed in the first $I_{\mathrm{CFD}}$ interval from a set of representative snapshots (using standard POD formulae to decide the number of retained modes). In subsequent $I_{\mathrm{CFD}}$ intervals, the POD manifold is only updated by applying POD to a set of vectors resulting from appropriately weighting old and new modes, namely the modes defining the former POD manifold and the modes resulting from applying POD to the newly calculated snapshots. Thus, the old and new POD modes (instead of the old and new snapshots) are appropriately mixed to update the POD manifold. This way of updating the POD manifold maintains the cost associated with the new snapshots calculation fairly small (a few of them are usually enough). As anticipated above, the inner product used to both apply POD and perform Galerkin projection is based on a limited number of mesh points. These points, as well as all other tunable parameters in the method, can be selected according to some simple, flexible criteria (e.g. they may be concentrated in areas of the domain where solutions exhibit a richer spatial structure, and chosen to avoid concentrated CFD errors). 

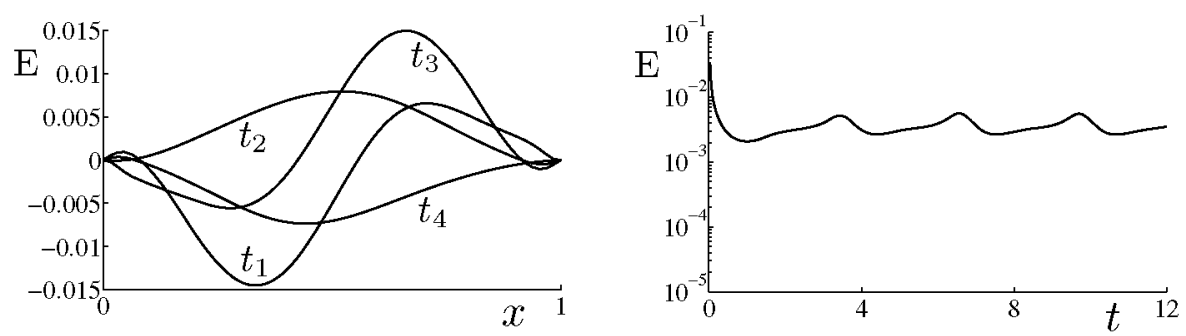

Figure 5. Errors of the CFD solution in a $64 \times 64$ mesh, at $R e=100$, with forcing $h(t)=\sin t$. Left: error in the nonhomogeneous upper boundary condition for the velocity, at four equally spaced values of $t$ along a forcing period. Right: time evolution of the relative rms error in the whole domain.

A key point of the LPOD+GP method consists in deciding when each $I_{\mathrm{GS}}$ interval must be terminated and the POD manifold updated by switching to a new $I_{\mathrm{CFD}}$ interval. This may be needed because either (i) the GS approximation by $n$ modes is no longer satisfactory (due to a change in the dynamics), which is detected using a standard a priori error estimate based on high-order modes, similar to its counterpart used in spectral methods for dealiasing (Gottlieb and Orszag 1977); or (ii) the current GS became unstable due to high-order modes truncation, which is anticipated using an estimate based on the comparison between the results provided by the two GSs, according to the remarks above.

For illustration, the method is applied here to the two-dimensional unsteady lid-driven cavity problem (Duck 1982, Ghia et al 1982, Schreiber and Keller 1983, Cazemier et al 1998, Ahlman et al 2002), which is regarded as a benchmark for closed laminar flows and describes the fluid motion in a squared cavity whose upper wall is moved back and forth by an external forcing. This is a fairly demanding test problem, as the unsteady shear forcing is produced in the upper boundary layer, which plays an essential role in the dynamics and increases the number of required POD modes. The governing incompressible Navier-Stokes equations depend on the Reynolds number $R e=u^{*} L^{*} / v$, where $u^{*}$ is the peak lid forcing velocity, $L^{*}$ is the width of the cavity, and $v$ is the kinematic viscosity, and on the unsteady forcing velocity, which is assumed to be $\boldsymbol{v}=(h(t) g(x), 0)$, where $x$ is the non-dimensional horizontal coordinate, $g(x)=16 x^{2}(1-x)^{2}$ smooths out the upper corners singularity, and $h(t)$ allows us to force the flow in a periodic or quasi-periodic fashion, yielding non-trivial dynamics at large time for moderate values of the Reynolds number.

In order to mimic industrial solvers, a fast CFD fractional-step code based on staggered grids is used, in which (i) some details remain unknown, (ii) accuracy is limited (figure 5) and (iii) some artifacts are introduced for numerical purposes. For instance, an unphysical Neumann boundary condition is imposed on the pressure and the following approximation is performed in momentum equations $I-\delta_{t}(2 R e)^{-1}\left(\partial_{x x}+\partial_{y y}\right) \simeq\left[I-\delta_{t}(2 R e)^{-1} \partial_{x x}\right][I-$ $\left.\delta_{t}(2 R e)^{-1} \partial_{y y}\right]$, where $I$ and $\delta_{t}$ stand for the unit operator and the time step, respectively; the latter approximation introduces a spurious boundary layer near the walls.

Using an industrial-like CFD solver means that the LPOD+GP method must compete with a fast code computing poorly accurate snapshots, which negatively influences the ROM performance. Thus, the question of whether the ROM should be constructed by projecting (onto the POD manifold) the exact governing equations or the CFD numerical scheme becomes an important issue (see Barone et al (2009) for a discussion on this topic). In order to emphasize the flexibility of the method, the exact governing equations (ignoring all CFD numerical artifacts) are projected and the resulting system of ODEs is integrated using a Crank-Nicolson scheme. Results are illustrated in figure 6 , where a part $(130 \leqslant t \leqslant 250)$ of 

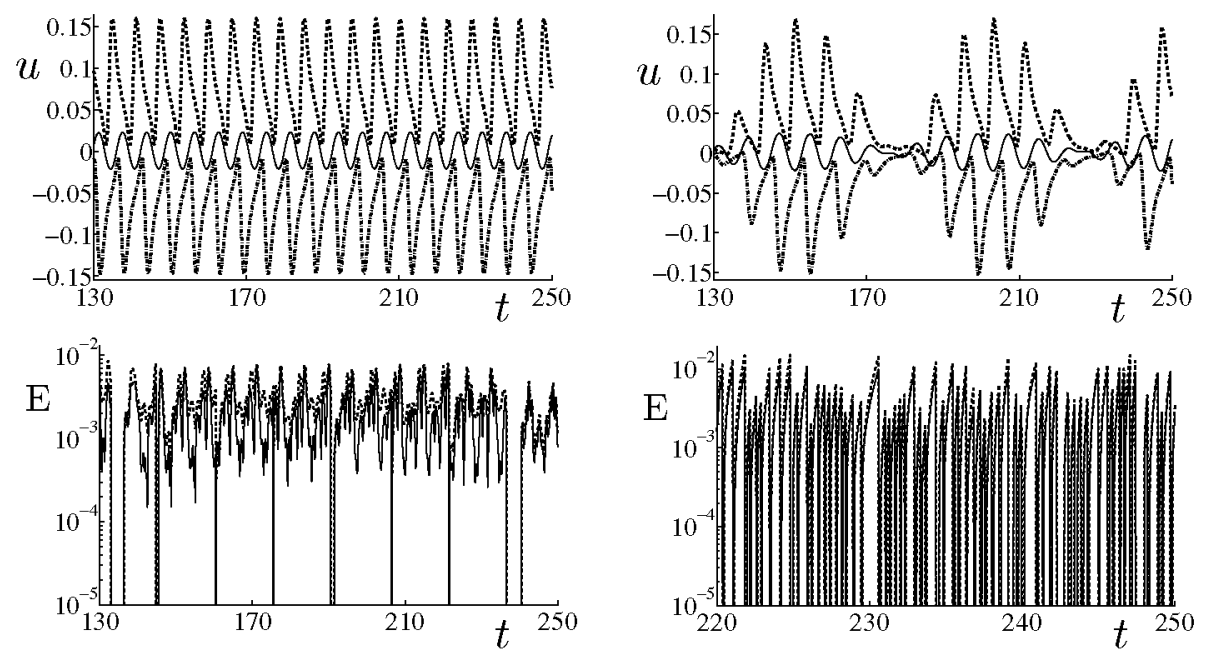

\begin{abstract}
Figure 6. Application of the LPOD+GP method to the flow at $R e=800$ with periodic $h(t)=\sin t$ (left) and quasi-periodic $h(t)=\sin (\pi t / 4) \cos (t / 16)$ (right) forcing. Top: time evolution of the horizontal velocity at two points near the left $(-\cdot-)$ and right $(--)$ upper corners, and the center of the cavity (-). Bottom: estimated (-) and exact (--) relative rms errors of the approximation provided by the ROM.
\end{abstract}

the transient behavior of the flow along the time span $0<t \leqslant 250$, from the quiescent state to the final attractor, at $R e=800$, is considered as excited by a periodic (left plots) and a quasi-periodic (right plots) forcing, which yields a periodic and a quasi-periodic response (as has been checked via Fourier transform) of the system (figure 6, top). The key observation for the good performance of the method is that the error estimate used by the method (figure 6 , bottom) to update the POD manifold works quite well, and that the required error bound, $\varepsilon=10^{-2}$, is close to the CFD solver accuracy (figure 5). This means, in particular, that the modes truncation instability has been eliminated by the method. Intrinsic instabilities of the flow are not present at these moderate values of the Reynolds number. The $I_{\text {CFD }}$ intervals can be identified in figure 6 (bottom) because the errors have been set to zero in these intervals, which are much smaller than the $I_{\mathrm{GS}}$ intervals. This is essential to obtain quite a good computational efficiency: the LPOD+GP ROM divides the CPU time required by the CFD solver by a factor of 9 and 4 in the periodic and quasi-periodic cases, respectively.

The application of the method to the complex Ginzburg-Landau equation (Aranson and Kramer 2002) by Rapún and Vega (2010) shows that it successfully approximates quite complex chaotic transient dynamics. Also, since the largest computational cost is associated with the calculation of the POD manifold in the first $I_{\mathrm{CFD}}$ interval, some libraries of modes can be used to further increase computational efficiency (Rapún et al 2011). Extensions to other fluid dynamics problems are currently under research, as is the combination of the method with parameter continuation, to obtain an adaptive, efficient means of constructing bifurcation diagrams in complex bifurcation problems.

\title{
4. Concluding remarks
}

Some basic ideas on reduced order modeling have been presented to construct generic, adaptive, robust, computationally efficient ROMs for both steady and unsteady fluid dynamics problems. In both cases, the ROM is constructed applying POD to a set of snapshots calculated 
with a rough CFD solver, similar to the ones that are usual in industry. The ROM construction method ignores numerical artifacts of the CFD solver, which enhances flexibility, and uses only a limited number of mesh points to project the governing equations and boundary conditions onto the POD manifold, which increases the computational efficiency.

In addition, concerning the application of steady ROMs to aerodynamic flows, the ROM is constructed using information from a few points in the O-mesh, near the wall, outside the boundary layers. The ROM is constructed using the Euler equations, ignoring both viscous effects and turbulence modeling artifacts. In spite of all these, the resulting ROMs approximate well the CFD results in the whole computational domain, including the wake and the boundary layers.

High-order modes truncation instability is monitored in unsteady ROMs and avoided by updating the POD manifold, which also allows for adapting the manifold to the local dynamics when needed. Thus, the ROMs approximate fairly well the transient dynamics in the unsteady lid-driven cavity problem.

For both steady and unsteady ROMs, the CPU time required by CFD is divided by a significant factor, maintaining a reasonable accuracy (of the order of that provided by the CFD solver).

Thus, we hope that the methods summarized in the paper will contribute to current efforts at developing computationally efficient ROMs to simulate complex fluid flows. These ROMs turn out to be quite efficient in approximating complex bifurcation diagrams involving steady, periodic, quasi-periodic and chaotic attractors. This has been the object of our recent research and will be considered elsewhere (Terragni and Vega 2012).

\section{Acknowledgments}

This work was partially supported by the Spanish Ministry of Education under grant no TRA2010-18054. We are indebted to an anonymous referee for comments on an earlier version of this paper that helped us to improve the presentation of the results.

\section{References}

Ahlman D, Söderlund F, Jackson J, Kurdila A and Shyy W 2002 Proper orthogonal decomposition for time-dependent lid-driven cavity flows Numer. Heat Transfer B $\mathbf{4 2} 285-306$

Alonso D, Velazquez A and Vega J M 2009a A method to generate computationally efficient reduced order models Comput. Methods Appl. Mech. Eng. 198 2683-91

Alonso D, Velazquez A and Vega J M 2009 b Robust reduced order modeling of heat transfer in a back step flow Int J. Heat Mass Transfer 52 1149-57

Alonso D, Vega J M and Velazquez A 2010 Reduced order model for viscous aerodynamic flow past an airfoil AIAA J. 48 1946-58

Alonso D, Vega J M, Velazquez A and de Pablo V 2012 Reduced order modeling of three dimensional, external aerodynamic flow J. Aerosp. Eng. in press

Aranson I S and Kramer L 2002 The world of the complex Ginzburg-Landau equation Rev. Mod. Phys. 74 100-42

Bache E, Vega J M and Velazquez A 2010 Model reduction in the back step fluid-thermal problem with variable geometry Int. J. Thermal Sci. 49 2376-84

Bache E, Alonso D, Velazquez A and Vega J M 2012 A computationally efficient reduced order model to generate multi-parameter fluid-thermal databases Int. J. Therm. Sci. 52 145-53

Barone M F, Kalashnikova I, Segalman D J and Thornquist H 2009 Stable Galerkin reduced order models for linearized compressible flow J. Comput. Phys. 288 1932-46

Bergmann M and Cordier L 2008 Optimal control of the cylinder wake in the laminar regime by trust-region methods and POD reduced-order models J. Comput. Phys. 227 7813-40

Bergmann M, Bruneau C H and Iollo A 2009 Enablers for robust POD models J. Comput. Phys. 228 516-38 
Berkooz G, Holmes P and Lumley J L 1993 The proper orthogonal decomposition in the analysis of turbulent flows Annu. Rev. Fluid Mech. 25 539-75

Bizon K, Continillo G, Russo L and Smula J 2008 On POD reduced models of tubular reactor with periodic regimes Comput. Chem. Eng. 32 1305-15

Cazemier W, Verstappen R W C P and Veldman A E P 1998 Proper orthogonal decomposition and low-dimensional models for driven cavity flows Phys. Fluids $101685-99$

Couplet M, Basdevant C and Sagaut P 2005 Calibrated reduced-order POD-Galerkin system for fluid flow modelling J. Comput. Phys. 207 192-220

Dowell E H and Hall K C 2001 Modeling of fluid-structure interaction Annu. Rev. Fluid Mech. 33 445-90

Duck P W 1982 Oscillatory flow inside a square cavity J. Fluid Mech. 122 215-34

Edwards J R and Chandra S 1996 Comparison of eddy viscosity transport turbulence models for three dimensional, shock separated flow fields AIAA J. 34 756-63

Ghia U, Ghia K N and Shin C T 1982 High-Re solutions for incompressible flow using the Navier-Stokes equations and a multigrid method $J$. Comput. Phys. 48 387-411

Gottlieb D and Orszag S A 1977 Numerical Analysis of Spectral Methods: Theory and Applications (Philadelphia: SIAM)

LeGresley P and Alonso J 2001 Investigation of non-linear projection for POD based reduced order models for aerodynamics ALAA Paper AIAA-2001-0926

Lieu T, Farhat C and Lesoinne M 2006 Reduced-order fluid/structure modeling of a complete aircraft configuration Comput. Methods Appl. Mech. Eng. 195 5730-42

Lucia D J, Beran P S and Silva W A 2004 Reduced-order modelling: new approaches for computations physics Prog. Aerosp. Sci. 40 51-117

Meis M, Varas F, Velázquez A and Vega J M 2010 Heat transfer enhancement in microchannels caused by vortex promoters Int. J. Heat Mass Transfer $5329-40$

Noack B R, Afanasiev K, Morzynski M, Tadmor G and Thiele F 2003 A hierarchy of low-dimensional models for the transient and post-transient cylinder wake J. Fluid Mech. 497 335-63

Rapún M L and Vega J M 2010 Reduced order models based on local POD plus Galerkin projection J. Comput. Phys. $2293046-63$

Rapún M L, Terragni F and Vega J M 2011 Mixing snapshots and fast time integration of PDEs Proc. IV Int. Conf. on Comput. Methods for Coupled Problems in Science and Eng. ed M Papadrakakis, E Oñate and B Schrefler (Coupled Problems, Article no. 246) pp 1-12

Ravindran S S 2000 Reduced-order adaptive controllers for fluid flows using POD SIAM J. Sci. Comput. 15 457-78

Rempfer D 2000 On low-dimensional Galerkin models for fluid flow Theor. Comput. Fluid Dyn. 14 75-88

Rempfer D 2003 Low-dimensional modeling and numerical simulation of transition in simple shear flows Annu. Rev. Fluid Mech. $35229-65$

Rouizi Y, Favennec Y, Ventura J and Petit D 2009 Numerical model reduction of 2D steady incompressible laminar flow: application on the flow over a backward facing step J. Comput. Phys. 228 2239-55

Schreiber R and Keller H B 1983 Driven cavity flows by efficient numerical techniques J. Comput. Phys. 49 $310-33$

Siegel S G, Cohen K and McLaughlin T 2003 Feedback control of a circular cylinder wake in experiment and simulation AIAA Paper AIAA-2003-3569

Siegel S G, Seidel J, Fagley C, Luchtenburg D M, Cohen K and McLaughlin T 2008 Low-dimensional modelling of a transient cylinder wake using double proper orthogonal decomposition J. Fluid Mech. $6101-42$

Sirisup S and Karniadakis G E 2004 A spectral viscosity method for correcting the long-term behavior of POD models J. Comput. Phys. 194 92-116

Sirisup S, Karniadakis G E, Xiu D and Kevrekidis I G 2005 Equations-free/Galerkin-free POD assisted computation of incompressible flows $J$. Comput. Phys. 207 568-87

Sirovich L 1987 Turbulence and the dynamics of coherent structures Q. Appl. Math. XLV 561-90

Spalart P R and Allmaras S R 1992 A one-equation turbulence model for aerodynamic flows AIAA Paper AIAA-920439

Tadmor G, Lehmann O, Noack B R and Morzynski M 2010 Mean field representation of the natural and actuated cylinder wake Phys. Fluids 22034102

Tannehill J C, Anderson D A and Pletcher R H 1997 Computational Fluid Mechanics and Heat Transfer (London: Taylor and Francis)

Terragni F, Valero E and Vega J M 2011 Local POD plus Galerkin projection in the unsteady lid-driven cavity problem SIAM J. Sci. Comput. 33 3538-61

Terragni F and Vega J M 2012 On the use of POD-based ROMs to analyze bifurcations in dissipative systems, preprint

Thomas J P, Dowell E H and Hall K C 2010 Using automatic differentiation to create a nonlinear reduced-order-model aerodynamic solver AIAA J. 48 19-24 